\title{
Interactive Editing of 3D Garment Using the Hyperquadratic Surface
}

\author{
Hongli DUN ${ }^{1}$, Ye YUAN*,Mengiiao DONG ${ }^{2}$ \\ School of Information Engineering, Beijing Institute of Fashion Technology, Beijing, China \\ dunhongli123@163.com,gxyyy@bift.edu.cn,1565315241@qq.com
}

\begin{abstract}
In view of the key technical problems that need to be solved in the digitization of 3D garments, this paper mainly studies the interactive editing of $3 \mathrm{D}$ garment surfaces. Therefore, this paper proposes a 3D garment surface interactive editing based on hyperquadratic surfaces. Firstly, the parametric representation of the hyperquadric surface of the editing region is obtained. Then the local editing of the surface is realized by changing the parameters of the super quadratic de-face. Finally, the algorithm of the waist piece is selected to verify the algorithm. The simulation results show that the proposed algorithm has certain effectiveness.
\end{abstract}

Keywords—surface editing, hyperquad surface, parameterization, $3 \mathrm{D}$ pieces

\section{INTRODUCTION}

After the general shape of the garment is determined, it is still necessary to make some detail adjustments, so that the modeling effect is colorful and more personalized, which requires partial editing of the $3 \mathrm{D}$ garment surface. The selected target surface needs to be able to achieve 2D expansion after editing. Superquadratic surfaces are widely used in $3 \mathrm{D}$ surface reconstruction problems ${ }^{[1,2,3]}$. In this paper, 3D modeling of local regions is performed by using hyperquadratic surfaces, and the surface is deformed by changing the coefficients of the hyperquadric surfaces to achieve partial editing of the surface. the goal of. The description of the surface by the hyperquadric surface reflects the super expressive power. It can realize the representation of various objects by controlling a small number of coefficients, and its description of the object shows extremely stable characteristics, so in computer graphics, the field of computer vision has been widely used.

\section{SUPER QUADRATIC SURFACES}

Superquadratic surfaces ${ }^{[4,5]}$ can be extended to four types of models: superellips, superrings, super-single hyperboloids, and super-double-leaf hyperboloids, of which only superellips are non-porous fully enclosed surfaces, so the space used to represent objects is sometimes used. The super ellipse is represented.

The implicit function of the hyperquadratic surface of the regular position is expressed as in formula (1).

$f\left(x_{v}, y\right)=\left[\left(\frac{x}{a}\right)^{\frac{2}{x^{2}}}+\left(\frac{y}{b}\right)^{\frac{2}{n^{2}}}\right]^{\frac{s^{2}}{s^{1}}}+\left(\frac{z}{e^{2}}\right)^{\frac{2}{s^{1}}}-1=0$

The a, b, and c sub-tables indicate the axial length of the hyperquadratic surface in the $\mathrm{x}, \mathrm{y}$, and $\mathrm{z}$ directions, $\mathrm{s} 1$ and $\mathrm{s} 2$ are deformation parameters, and s1 controls the degree of deformation of the hyperquadric surface in the $\mathrm{x}$ and $\mathrm{y}$ direction, and $\mathrm{s} 2$ controls the super degree of deformation of the quadric surface in the $\mathrm{z}$ direction. These five parameters together determine the final shape of the hyperquadric surface. In order to increase the expressive ability of the hyperquadratic surface to any surface, the transformation of the hyperquadratic surface is controlled by the translation and rotation of the coordinates of the hyperquadric surface. Assume that any point like $p(x, y, z)^{T}$ on the super quadratic surface is tapered along the $\mathrm{z}$-axis to obtain a point that is $p_{\text {tapey }}=\left(x_{\text {tapey }} y_{\text {tapey }}, z_{\text {tapey }}\right)^{T}$. The relationship between the two is as shown in formula (2). 


$$
p_{\text {tapey }}=f_{\text {tapey }}(p)=\left(\left(\frac{k_{x} z}{c}\right) x_{v}\left(\frac{k_{y} z}{c}\right) y_{s} z\right)^{T}
$$

the taper deformation function is expressed, $k_{x}$ and $k_{y}$ the taper deformation coefficient is satisfied.

Among them, $f_{\text {Taper }}(*)$ denotes a taper deformation function, $k_{x}$ and $k_{y}$ denote a taper deformation coefficient satisfying $-1 \leq k_{x}, k_{y} \leq 1$

By adding translation and rotation transformation to the hyperquadric surface after the taper deformation, the pose of the hyperquadric surface can be arbitrarily transformed. In order to better describe the translation and rotation transformation of the hyperquadratic surface, the parametric equation of the superquadratic surface is introduced, as in formula (3).

$\left\{\begin{aligned} x=a \cdot \sin ^{32} \omega \cos ^{31} \eta \\ y=b \cdot \cos ^{32} \omega \cos ^{21} \eta,-\frac{\pi}{2} \leq \eta \leq \frac{\pi}{2},-\pi \leq \omega \leq \pi \\ \quad z=c \cdot \sin ^{31} \eta\end{aligned}\right.$

Suppose $p_{W}=\left(x_{w^{w}} y_{W^{x}} z_{W}\right)^{T}$ is the corresponding point after $p_{\text {tapey }}$ is translated and rotated, then there is $p_{w}=R \cdot p_{\text {tap }}+t$.

Among them, the calculation formula of the rotation matrix and the translation variable is as shown in formula (4).

$\mathbb{R}=f\left(\varphi_{x}, \theta_{\alpha} \psi\right)=$

[cos $\varphi \cos 9 \cos \varphi \sin 9 \sin \psi-\sin \varphi \cos \psi \mathrm{cos} \varphi \sin \theta \cos \varphi+\sin \varphi \sin \psi]$ $\left[\begin{array}{ccc}\sin \varphi \cos \theta & \sin \varphi \sin \theta \sin \psi+\cos \varphi \cos \psi & \sin \varphi \sin \theta \cos \psi-\cos \varphi \sin \psi \\ -\sin \theta & \cos \theta \sin \psi & \cos \theta \cos \psi\end{array}\right]$
Among them, $\varphi$ is the roll angle, $\theta$ is the pitch angle, $\psi$ is the deflection angle, and the value ranges are respectively $\varphi \in[-\pi, \pi], \theta \in\left[-\frac{\pi}{2}, \frac{\pi}{2}\right], \psi \in\left[-\frac{\pi}{2}, \frac{\pi}{2}\right]$.

The translation vector is $t=\left(t_{x,}, t_{y}, t_{z}\right)^{T}$, so the model parameter $A$ of the hyperquadric of any pose can be described as: $A=\left\{a, b, c_{x} s 1_{s} s 2_{x} k_{x}, k_{y}, \varphi, \theta, \psi, t_{x,}, t_{y}, t_{z}\right\}$

When the superquadratic parameter model only adds the axial length parameter and the deformation parameter, the parameter expression of the hyperquadric surface is in (3). It is assumed that the initial values of each parameter of the hyperquadratic parameter equation are all one. Following the principle of single variable, the parameters of the hyperquadratic surface are controlled to realize the deformation of the hyperquadric surface. As shown in Fig.1 and Fig.2.

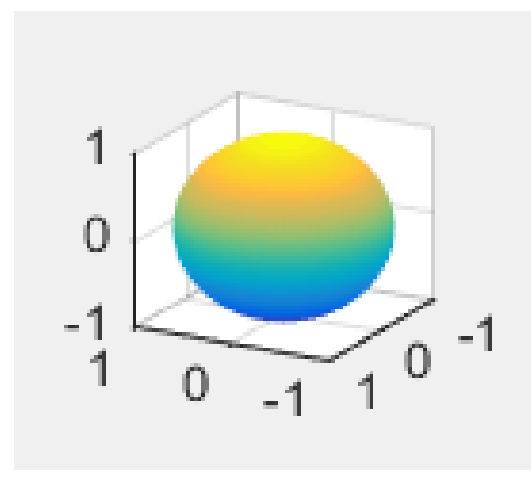

Fig.1. parameters are the corresponding spherical surface at initialization

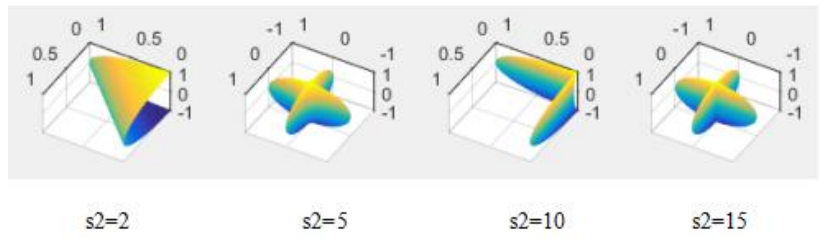

Fig.2. Shows the corresponding surface shape after changing a certain control parameter surface pretreatment 
To edit the selected area to be edited, you first need to fit with a hyperquadric surface to get the best representation of the surface and determine the expression of the surface ${ }^{[6]}$. By parameterizing the edited surface, multiple control parameters are obtained. Then, by adjusting the values of the plurality of control parameters, the free deformation of the 3D surface is achieved. In order to fit the surface of the surface to be edited, the function is defined as in (5).

$$
F(x, y, z)=(f(x, y, z)-1)^{\varepsilon}
$$

Set any point $p(x, y, z)$ on the surface. When

$F(x, y, z)>1$, the point is outside the hyperquadric surface.

When $F\left(x_{v} y_{x} z\right)<1$, the point is inside the hyperquadric

surface. When $F(x, y, z)=1$, the point is on the hyperquadric surface. The fitting of a hyperquadratic surface is a problem of nonlinear fitting, and it is necessary to set an initial value for a parameter. The commonly used least squares algorithm for parameter fitting method of super quadratic surfaces is used. The optimization algorithms include L-M algorithm, particle swarm optimization algorithm and invasive weed optimization algorithm.

\section{A. Selection of editing area}

To achieve partial editing of a triangular mesh surface, you must first obtain the area to be edited. According to the construction method of the surface expansion, in order to obtain the editing area, it is necessary to first determine the most value point on the boundary of the area to be edited, that is, the corner point with the editing area. The included triangle mesh is then determined by the extents defined by the four corner points and the vertices of the triangular mesh are determined.

\section{B. Editing area parameterization}

After the editing area is determined, the vertices of all the triangle meshes contained in the editing area will be obtained. Then the surface of the hyperquadric surface is fitted by the vertices of these triangular meshes to realize the parameterization of the area to be edited. The specific process of parameterization is elaborated in the surface preprocessing. After obtaining the parametric representation of the hyperquadratic surface of the point editing area, the local editing of the surface is realized by changing the parameters of the super quadratic de-surface.

Taking the hyperquadric surface with only the axial length and deformation parameters as an example, the fitting algorithm of the hyperquadric surface is as follows:

Step1: Set the initialization equation and initialization parameters of the hyperquadratic surface. It includes axis length parameters a, b, c and deformation parameters s1, s2.

Step2: Get the vertices of all triangle meshes in the editing area.

Step3: Change the parameters of the hyperquadratic surface, and find the least square difference of the distance between the hyperquadratic surface and the area to be edited by equation (5). In order to improve the efficiency, only the last set of parameters that minimize the variance of the distance is saved. The value of $x_{s} y_{s} z$ in equation (5) takes the vertex coordinates of all triangular meshes in Step 2.

Step4: Stop the fitting of the hyperquadratic surface when the least square difference of the distance reaches a certain threshold.

\section{Partial editing of $3 D$ pieces}

The local editing implementation algorithm of the 3D piece is as follows:

Step1: Select the surface to be edited and get the vertices of the triangle mesh inside the surface.

Step2: Perform super quadratic surface fitting on the edited area, and obtain a parametric representation of the hyperquadric surface of the area to be edited.

Step3: Local editing of the surface by changing the parameters of the hyperquadric surface.

Step4: For the partially edited surface, re-expand 2D by the method of Chapter 3. For the surface adjacent to the surface, if the boundary points of the two surfaces change, 
the surface needs to be connected to the curved surface. The surface regenerates the $2 \mathrm{D}$ expansion.

\section{EXPERIMENTAL RESULTS AND ANALYSIS}

In order to verify the feasibility and effect of the algorithm, the experiment selected the waist piece to apply this algorithm for local editing. The $2 \mathrm{D}$ expansion of the piece before editing is shown in Figure 3, and the 2D expansion of the piece after editing is shown in Figure 4:
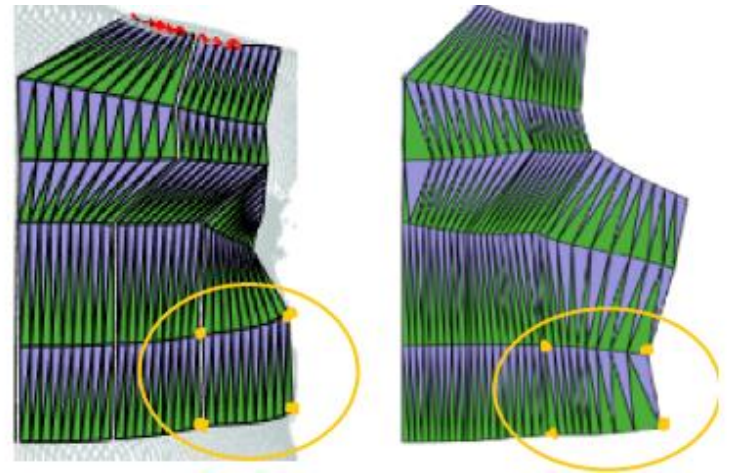

Fig.3. 2D expansion of the piece before editing
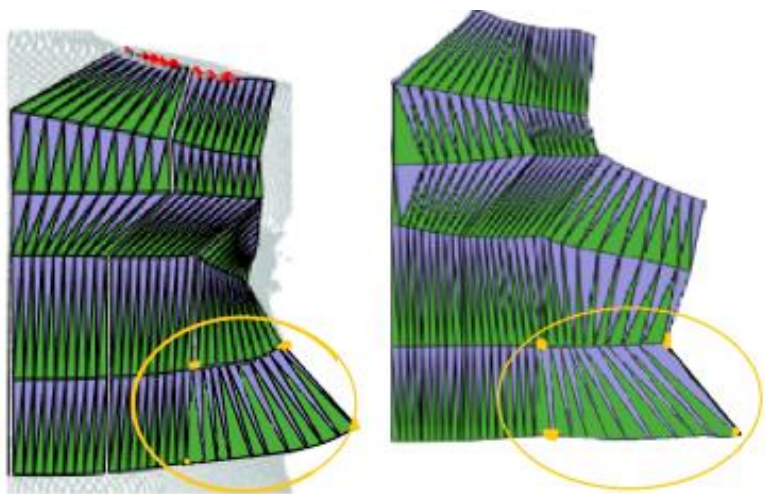

Fig.4. 2D expansion of the piece after editing

Comparing the surface before and after partial editing, it is known that the boundary point needs to be re-acquired when performing local editing through the hyperquadric surface. For the boundary point where the change is generated, the surface generated by the control needs to be re-expanded 2D.

\section{CONCLUSION}

The partial editing of the surface is an important aspect of the surface modeling. In the $3 \mathrm{D}$ garment design, it is decisive for the detailed processing of the garment, the style of the garment and the final molding effect. To use the hyperquadric surface to perform local editing of the target surface, only the surface of the super quadratic surface is fitted to the target surface. After obtaining the parametric equation of the hyperquadratic surface, the parameters of the hyperquad surface can be adjusted. Applying deformation to the target surface has the advantage of being simple in algorithm. The experimental results show that the algorithm performs local editing on the target surface, and the effect is obvious and easy to control.

\section{ACKNOWLEDGEMENT}

This work is supported by the science and technology project of Beijing Municipal Education Commission of 2017 with the granted No. SQKM201710012007 and also by the "Textile light" China Textile Industry Federation education and teaching reform project of 2017 with the granted No. 2017BKJGLX219 .

\section{REFERENCES}

[1] Kazhdan M, Bolitho M, Hoppe H. Poisson surface reconstruction[C].Eurographics Symposium on Geometry Processing. 2006:61-70.

[2] Kazhdan M, Hoppe H. Screened poisson surface reconstruction[J] Acm Transactions on Graphics, 2013, 32(3):1-13.

[3] Gopi M, Krishnan S, Silva C T. Surface Reconstruction based on Lower Dimensional Localized Delaunay Triangulation[J]. Computer Graphics Forum, 2010, 19(3):467-478.

[4] Solina F, Bajcsy R. Recovery of parametric models from range images: The case for superquadrics with global deformations[J]. IEEE Transactions on Pattern Analysis and Machine Intelligence, 1990, 12(2): 131-147.

[5] Staglianò G. On special quadratic birational transformations of a projective space into a hypersurface[J]. Rendiconti Del Circolo Matematico Di Palermo, 2012, 61(3):403-429.

[6] Solomon N, Zhang R. Highly incidental patterns on a quadratic hypersurface in R 4 R 4 math Container Loading Mathjax, [J]. Discrete Mathematics, 2016, 340(4):585-590. 